\title{
BURNOUT: O IMPACTO DA SATISFAÇÃO NO TRABALHO EM
} PROFISSIONAIS DE ENFERMAGEM

\author{
BURNOUT: EL IMPACTO DE LA SATISFACCIÓN EN EL TRABAJO EN LOS \\ PROFESIONALES DE ENFERMERÍA \\ BURNOUT SYNDROME: THE IMPACT OF JOB SATISFACTION AMONG \\ NURSING PERSONNEL
}

\author{
Adriana Müller Saleme de Sá \\ Universidade de Vila Velha, Vila Velha/ES, Brasil \\ Priscilla de Oliveira Martins-Silva \\ Universidade Federal do Espírito Santo, Vitória/ES, Brasil
}

Bruno Funchal

FUCAPE Business School, Vitória/ES, Brasil

\begin{abstract}
RESUMO
O objetivo do artigo é analisar a relação entre fatores de satisfação no trabalho (satisfação com as relações hierárquicas; satisfação com o ambiente físico do trabalho; satisfação com o trabalho e oportunidade de crescimento) e dimensões da Síndrome de Burnout (exaustão emocional, despersonalização e realização profissional) em profissionais de enfermagem de um hospital público. Utilizou-se a abordagem quantitativa. A amostra foi composta por 52 enfermeiros. Os instrumentos aplicados foram: questionário sociodemografico, Maslach Burnout Inventory, Questionário de Satisfação no Trabalho S20/23. Para a análise utilizou-se a regressão linear múltipla dos Mínimos Quadrados Ordinários. Os resultados apresentaram que o fator satisfação com o ambiente reduz a exaustão emocional; o aumento com a satisfação com o trabalho e oportunidade de crescimento reduz as dimensões exaustão emocional e despersonalização; e também aumenta a realização profissional.
\end{abstract}

Palavras-chave: Síndrome de Burnout; satisfação no trabalho; enfermagem.

\section{RESUMEN}

El objetivo de este artículo es analizar la relación entre los factores de satisfacción en el trabajo (satisfacción con las relaciones jerárquicas, la satisfacción con el ambiente físico de trabajo, la satisfacción laboral y la oportunidad de crecimiento) y las dimensiones del Síndrome de Burnout (agotamiento emocional, despersonalización y logro profesional) en profesionales de enfermería de un hospital público. Se utilizó un enfoque cuantitativo. La muestra fue compuesta por 52 enfermeras. Los instrumentos utilizados fueron: cuestionario sociodemográfico, Maslach Burnout Inventory, el Cuestionario de Satisfacción S20/23. Para el análisis se utilizó la regresión múltiple de mínimos cuadrados ordinarios. Los resultados presentaron que lo factor de satisfacción con el entorno reduce el cansancio emocional; aumenta la satisfacción laboral y la oportunidad de crecimiento reducen el cansancio emocional y despersonalización y también aumenta el logro profesional.

Palabras clave: Síndrome de Burnout; satisfacción en el trabajo; enfermería.

\begin{abstract}
This paper aims to analyze the relationship between job satisfaction factors (hierarchy relations satisfaction, physical ambience satisfaction and work and growth opportunity satisfaction) and dimensions of Burnout Syndrome (emotional exhaustion, depersonalization, and professional accomplishment) for nurses in a public hospital. In this paper we use a quantitative approach, applying the Maslach Burnout Survey and Job Satisfaction Survey S20/23, together with social-demographic information on a sample of 52 nurses. Using the Ordinary Least Squares multiple linear regression as the empirical method, we found that physical ambience satisfaction reduces emotional exhaustion, and an increase in work and growth opportunity satisfaction reduces the emotional exhaustion and also increases professional accomplishment.
\end{abstract}

Keywords: Burnout Syndrome; job satisfaction; nursing. 


\section{Introdução}

O objetivo deste artigo consiste em analisar a relação entre os fatores de satisfação no trabalho e a Síndrome de Burnout em profissionais de enfermagem em um hospital público da Região Sudeste. Especificamente, tem-se como objetivo testar a relação entre os fatores de satisfação no trabalho com as dimensões da Síndrome de Burnout (Exaustão Emocional, Despersonalização e Realização Profissional) (Maslach \& Jackson, 1981).

Além de identificar os determinantes de cada dimensão da síndrome, este artigo contribui em termos metodológicos. Diferentemente da literatura atual (Moreira, Magnago, Sakae, \& Magalewski, 2009; Rosa \& Carlotto, 2005; Ruviaro \& Bardagi, 2010; Silva \& Carlotto, 2008), é utilizado para análise dos dados o procedimento de regressão linear múltipla. Nos estudos citados é utilizado o procedimento de correlação bivariada, procedimento que não permite identificar a sensibilidade de cada dimensão da Síndrome de Burnout a seus possíveis determinantes de forma conjunta.

A categoria profissional de enfermagem é marcada por componentes ameaçadores do ambiente ocupacional, tais como o número reduzido de profissionais para fazer o atendimento em saúde, o excesso de atividades que executa e a falta de reconhecimento (Stacciarini \& Tróccoli, 2001), além de o hospital ser reconhecido como um ambiente insalubre, penoso e perigoso (Elias \& Navarro, 2006). Observa-se também a questão dos baixos salários que os obriga a ter mais de um emprego, resultando em longa e desgastante jornada mensal de trabalho (Murofurose, Abranches, \& Napoleão, 2005).

Não são somente os aspectos operacionais que tornam o trabalho de enfermagem desgastante. Observam-se também as exigências relativas à responsabilidade para com seus pacientes, tanto no aspecto físico quanto no aspecto moral, social e psicológico, além do fato da enfermagem possuir pouco status e prestígio numa organização (Dias, Boas, Dias, \& Barcellos, 2005). Outro fator é o contato direto com a dor, o sofrimento e a morte, o que exige desse profissional um controle emocional maior se comparado a outras profissões (BenevidesPereira, 2002). É nesse contexto que Silva, Lima, Farias e Campos (2006, p. 442) afirmam que "os eventos estressantes permeiam os hospitais e levam os enfermeiros, bem como os demais profissionais, ao esgotamento, gerador de profissionais indiferentes, apáticos e cansados, dominados por estresse e desmotivação, com consequentes conflitos e insatisfações".

Portanto, quando essas situações ocupacionais são persistentes, é possível o desenvolvimento da Síndrome de Burnout nesses profissionais. Neste estudo, será utilizada a definição de Síndrome de Burnout (SB) proposta por Maslach e Jackson (1981) por ser considerada uma das definições mais aceitas na literatura (Carlotto, 2002; Carlotto \& Câmara, 2008a). De acordo com Maslach e Jackson (1981), a SB caracteriza-se pela presença de exaustão emocional, despersonalização e baixa realização profissional que ocorrem frequentemente em pessoas que executam algum tipo de trabalho em que precisem se relacionar com pessoas de forma próxima e direta.

$\mathrm{Na}$ Exaustão Emocional, o trabalhador se sente esgotado, com forças diminuídas e exaurido de recursos físicos e emocionais, com dificuldades para enfrentar mais um dia de trabalho e queixando-se frequentemente de estar sobrecarregado e assoberbado. A Exaustão Emocional é considerada a qualidade central da SB e a manifestação mais óbvia dessa complexa síndrome, pois, se uma pessoa se descreve experimentando a SB, ela estará, frequentemente, referindo-se à experiência de exaustão (Maslach, Schaufeli, \& Leiter, 2001). A exaustão, geralmente, pode ser descrita como oriunda da sobrecarga de trabalho e do conflito pessoal no trabalho (Maslach, 2005; Wright \& Bonett, 1997).

A Despersonalização é uma tentativa de colocar distância entre si e as pessoas com quem se relaciona no trabalho, como os clientes e os colegas. Nessa situação, o distanciamento é uma reação imediata à exaustão, havendo uma forte relação com esse fator (Maslach et al., 2001). A Despersonalização refere-se ao desenvolvimento de atitudes negativas e insensíveis para com as pessoas com quem se trabalha.

Com o tempo, à medida que a Despersonalização vai progredindo, os trabalhadores reduzem a quantidade de tempo que passam no trabalho e a quantidade de energia despendida na realização do mesmo, interferindo na qualidade de seu desempenho. Consequentemente, o indivíduo passará a ter sensações de incompetência, falta de realização e produtividade no trabalho, que é a terceira dimensão da síndrome, a Realização Profissional diminuída (Maslach, 2005). A Realização Profissional diminuída parece surgir de forma mais clara a partir da falta de recursos relevantes, enquanto a Exaustão Emocional e a Despersonalização derivam da sobrecarga de trabalho e dos conflitos sociais (Maslach et al., 2001).

Pode-se dizer então que a Síndrome de Burnout é uma mistura de um estado individual (Exaustão 
Emocional), de uma estratégia de enfrentamento (Despersonalização) e de uma consequência (Realização Pessoal diminuída), que podem ser estudadas conjuntamente ou separadamente (Schaufeli \& Taris, 2005).

Embora existam outras definições acerca da SB, observam-se pelo menos cinco elementos comuns entre todas as definições. São eles: (a) predominância de sintomas relacionados à exaustão mental e emocional, fadiga e depressão; (b) maior presença de sintomas comportamentais, e não de sintomas físicos; (c) manifestação em pessoas que não sofriam de distúrbios psicopatológicos; (d) o surgimento dos sintomas estão relacionados ao trabalho; (e) diminuição da efetividade e do desempenho no trabalho decorrente de atitudes e comportamentos negativos (Maslach \& Schaufeli, 1993).

Para maior compreensão da SB entre os profissionais de enfermagem, os pesquisadores têm procurado estudar a relação da SB com determinadas variáveis. Geralmente verificam-se estudos associando a SB com variáveis sociodemográficas e com variáveis relacionadas ao mundo do trabalho (Moreira et al., 2009; Rosa \& Carlotto, 2005; Silva \& Carlotto, 2008). Os achados mais recentes nos estudos brasileiros sobre a relação de variáveis sociodemográficas e a SB têm encontrado que a idade está associada negativamente com as dimensões Exaustão Emocional (Moreira et al., 2009; Rosa \& Carlotto, 2005) e Despersonalização (Rosa \& Carlotto, 2005), indicando que, quanto mais novo o indivíduo, maior a propensão de desenvolver essas duas dimensões da síndrome. Além disso, verificase que profissionais com curso superior completo apresentam maiores índices de Despersonalização (Silva \& Carlotto, 2008). Isso pode ser explicado por esses profissionais poderem ter maiores expectativas em relação às suas carreiras, expectativas que não se tornam compatíveis com o cotidiano de seu trabalho. Também foi encontrado que pessoas sem filhos e solteiras estão associadas negativamente à dimensão Exaustão Emocional (Moreira et al., 2009).

Os estudos brasileiros mais recentes sobre a $\mathrm{SB}$ em profissionais de enfermagem e a sua relação com o mundo do trabalho tem abordado os seguintes aspectos: tempo de profissão/ experiência, carga horária, cargo desempenhado, setor de trabalho, escala de trabalho, sobrecarga de trabalho, coleguismo, práticas organizacionais e satisfação com o trabalho. Os resultados encontrados indicam que o tempo de profissão/experiência em um determinado local está associado com a Despersonalização (Silva \& Carlotto, 2008), pois à medida que o profissional adquire maior domínio sobre o seu trabalho e sobre as suas relações no local de trabalho, menor é o sentimento de distanciamento. Outra variável associada à SB é a carga horária de trabalho. Essa variável também está associada negativamente à dimensão Exaustão Emocional, indicando que, provavelmente, quando um profissional tem uma carga horária menor, ele sofre maior pressão para realizar determinado número de atendimentos dentro dessa carga horária (Silva \& Carlotto, 2008). A variável sobrecarga de trabalho foi associada às três dimensões da síndrome, o que demonstra que as demandas do trabalho podem favorecer o desenvolvimento da síndrome (Tamayo, 2009). A variável ausência de coleguismo apareceu associada à Exaustão Emocional e à diminuição da Realização Profissional (Tamayo, 2009). A variável práticas organizacionais apareceu associada à Exaustão Emocional (Tamayo, 2009), indicando que o tipo de tratamento que a organização dá aos seus profissionais interfere no desenvolvimento da Exaustão Emocional. Nas variáveis cargo desempenhado, setor de trabalho (Moreira et al., 2009), tempo de profissão (Moreira et al., 2009; Rosa \& Carlotto, 2005; Silva \& Carlotto, 2008) e escala de trabalho (Rosa \& Carlotto, 2005) não foram encontradas associações estatísticas significativas.

Três estudos brasileiros, nos últimos 5 anos, procuraram associar o nível de satisfação e a SB em profissionais de enfermagem. O primeiro trabalho (Silva \& Carlotto, 2008) verificou que a Exaustão Emocional associa-se negativamente às seguintes dimensões do conceito de satisfação: satisfação com as relações hierárquicas, satisfação com o trabalho intrínseco e satisfação com oportunidade de crescimento profissional. Também observou que a Realização Profissional associa-se positivamente a essas mesmas dimensões e que a Despersonalização está associada negativamente apenas à dimensão satisfação com as relações hierárquicas. O segundo trabalho (Ruviaro \& Bardagi, 2010) identificou que a Exaustão Emocional está associada com baixos índices de satisfação em relação ao salário, à tarefa, à promoção e à satisfação total; e a Realização Profissional está associada à satisfação com os colegas, com o salário, com a tarefa, com a promoção e com a satisfação geral. Não foram identificadas correlações entre Despersonalização e fatores de satisfação. O terceiro estudo (Neves, 2012) verificou que Exaustão Emocional, Despersonalização e Decepção no Trabalho estão associadas a baixos índices de satisfação com colegas, salário, chefia, trabalho, promoção e percepção de suporte organizacional. Além de verificar a correlação entre as variáveis estudadas, Neves (2012) também realizou análise de regressão múltipla (método stepwise) e verificou que, para a 
dimensão de Exaustão Emocional, há três variáveis preditoras: satisfação com a natureza do trabalho, percepção de suporte organizacional e satisfação com o salário; para a dimensão Despersonalização, há dois preditores: satisfação com a natureza do trabalho e percepção de suporte organizacional e; a dimensão Decepção no Trabalho apresentou três preditores: a satisfação com a natureza do trabalho, percepção de suporte organizacional e satisfação com a promoção.

Os dois primeiros estudos utilizaram em suas metodologias análises inferenciais: correlação de Pearson, teste t e ANOVA. O terceiro estudo, além de usar essas metodologias, também utilizou análise de regressão linear múltipla. Verifica-se que a utilização de regressão linear múltipla ainda é incipiente na literatura sobre SB, contudo este tipo de análise é mais rico que a análise de correlação bivariada por permitir a análise conjunta de diversas variáveis, possibilitando, assim, maior consistência na análise dos dados.

Resultados da literatura evidenciam a relação entre a SB e a satisfação no trabalho. As evidências demonstram que a SB está associada a uma menor satisfação no trabalho. O estudo da SB em profissionais de enfermagem é relevante, pois indivíduos com essa síndrome podem exercer impacto negativo sobre os seus colegas, tanto por causa dos conflitos pessoais quanto na interrupção de suas tarefas, o que pode fazer com que a SB seja contagiosa e se perpetue por meio de interações informais no trabalho, ou seja, a partir das interações entre os profissionais, por meio de comentários negativos e comportamentos inadequados para um ambiente de trabalho harmonioso (Maslach et al., 2001). Pode-se dizer, portanto, que a SB é uma experiência individual que prejudica a relação do indivíduo com seu trabalho, atrapalhando seu desempenho profissional, o que reflete em prejuízos para o indivíduo, para a organização e pode estenderse para o usuário do serviço (Benevides-Pereira, 2002; Oliveira, Tristão, \& Neiva, 2006).

Diante desses achados, observa-se que as condições de trabalho dos profissionais de saúde têm influência significativa tanto na qualidade de vida no trabalho quanto na qualidade do serviço prestado pelas organizações de saúde. As taxas de acidente de trabalho, doenças e absenteísmo entre os profissionais dessa área estão relacionadas às suas condições de trabalho. Por isso, é necessário que as organizações se preocupem em oferecer a seus empregados maior qualidade de vida no trabalho, pois o bem-estar e a saúde ocupacional gerarão impactos sobre a organização e também implicações para a sociedade em geral (Gil-Monte, 2001).
Apesar da Síndrome de Burnout ter sido incluída desde 1996 no Anexo II da Previdência Social como uma doença referente aos Agentes Patogênicos de Doenças Profissionais, ainda hoje ela é uma doença desconhecida da maioria das pessoas, tanto das que fazem parte do grupo de risco de desenvolver a síndrome quanto dos profissionais que "deveriam conhecê-la o suficiente para poder orientar, diagnosticar ou encaminhar para uma intervenção" (BenevidesPereira, 2003, pp. 4-5). Essa realidade é reflexo da produção nacional sobre o tema, que ainda é escassa. Em um levantamento realizado por Carlotto e Câmara (2008a), foram observados 28 artigos empíricos no período de sete anos. O primeiro artigo brasileiro encontrado foi no ano de 1999. Desses artigos, apenas sete abordam os profissionais de saúde. Diante do contexto em que se encontram as pesquisas sobre a SB no âmbito da literatura nacional e do impacto dessa síndrome nas pessoas, no ambiente de trabalho e na qualidade do trabalho verifica-se a relevância de aprofundar o seu conhecimento.

\section{Metodologia}

A presente pesquisa utilizou a abordagem quantitativa com a utilização de questionários com o objetivo de estudar a relação entre variáveis (Cozby, 2003). Este estudo foi realizado em um hospital público, situado na região Sudeste. A escolha do local para a realização deste estudo deu-se pelo fato da instituição prestar assistência de enfermagem 24 horas por dia e pelo fato de atenderem pacientes que necessitam de cuidados de enfermagem de nível mínimo, intermediário, semi-intensivo e intensivo. Como critério de inclusão para participar da pesquisa, os indivíduos deveriam atender aos seguintes quesitos: serem profissionais de enfermagem (enfermeiros, técnicos e auxiliares de enfermagem) que trabalham no referido hospital, independente do tempo de experiência na profissão ou setor de trabalho.

Para a coleta de dados foram utilizados três instrumentos: (a) questionário sociodemográfico, (b) Maslach Burnout Inventory (MBI) e (c) Questionário de Satisfação no Trabalho S20/23.

O questionário sociodemográfico é um questionário autoaplicável elaborado pelos pesquisadores com o objetivo de caracterizar o perfil sociodemográfico e a situação profissional da equipe de enfermagem.

Para avaliar a presença da Síndrome de Burnout, foi utilizado o Maslach Burnout Inventory (MBI), que é um inventário autoaplicável com 22 itens que avalia 
como o indivíduo vivencia seu trabalho, de acordo com as três dimensões estabelecidas pelo Modelo Teórico de Maslach: Exaustão Emocional - EE, Realização Pessoal no Trabalho - RP e Despersonalização - DE. Esse inventário teve sua tradução validada para a língua portuguesa por Benevides-Pereira (2001) e analisada em suas propriedades psicométricas (fidedignidade e validade de construto em uma amostra multifuncional de trabalhadores brasileiros) por Carlotto e Câmara (2007). Os resultados indicam consistência interna do instrumento em suas três dimensões ( $\mathrm{EE}, \alpha=0,88$; $\mathrm{RP}, \alpha=0,94$; $\mathrm{DE} \alpha=0,65)$. A dimensão $\mathrm{EE}$ tem sua confiabilidade de moderada a alta, porém esse resultado é um pouco superior ao encontrado pelas autoras do instrumento original (Maslach \& Jackson, 1981), que foi de $\alpha=0,60$.

Para avaliar a satisfação no trabalho foi utilizado o Questionário de Satisfação no Trabalho S20/23 de Meliá e Peiró (1989), validado e adaptado para o Brasil por Carlotto e Câmara (2008b). O instrumento adaptado e validado por Carlotto e Câmara (2008b) constitui-se de 20 itens que são divididos em três fatores (todos os fatores apresentaram $\alpha>0,77$ ): Satisfação com as Relações Hierárquicas - SRH, Satisfação com o Ambiente Físico de Trabalho - SAFT e Satisfação Intrínseca com o Trabalho e Oportunidades de Crescimento - SITOPC.

Todos os questionários foram entregues aos participantes para serem preenchidos individualmente. Depois de preenchidos, os questionários foram recolhidos.

Para identificar a associação entre as dimensões da Síndrome de Burnout e os fatores da satisfação no trabalho foi utilizado o método estatístico de regressão linear múltipla. Dentre as possibilidades desse método, optou-se pela técnica de Mínimos Quadrados Ordinários (MQO) para estimar as relações entre as variáveis definidas no modelo. Essa técnica apresenta uma contribuição nos estudos sobre SB uma vez que permite identificar os possíveis determinantes da $\mathrm{SB}$ de forma conjunta.

Para a realização do método de regressão linear múltipla dos MQO foram definidos três modelos empíricos. Foi utilizado o método padrão para a seleção das variáveis do modelo. O primeiro modelo empírico é a aplicação da regressão tendo, como variável dependente, a dimensão Exaustão Emocional (EE) da Síndrome de Burnout e, como variáveis independentes, os fatores da Satisfação no Trabalho (Satisfação com as Relações Hierárquicas - SRH, Satisfação com o Ambiente Físico do Trabalho - SAFT e Satisfação Intrínseca com o Trabalho e Oportunidade de Crescimento - SITOPC) e variáveis de controle (sexo, regime de contratação, categoria profissional, tempo de trabalho no setor e número de pessoas que atende). Nos demais modelos, foi aplicada regressão tendo as mesmas variáveis independentes e de controle, porém no segundo modelo utilizou-se como variável dependente a Despersonalização (DE) e no terceiro modelo utilizou-se como variável dependente a Realização Profissional (RP).

Este estudo foi realizado dentro dos padrões éticos de pesquisa e respeitou a Resolução 196/96, que regulamenta pesquisas envolvendo seres humanos, do Conselho Nacional de Saúde do Ministério da Saúde, e foi aprovado pelo Comitê de Ética e Pesquisa em Seres Humanos.

\section{Resultados}

Foram respondidos 56 questionários, porém quatro questionários foram invalidados por falta de informação nos mesmos. Portanto, fazem parte desta pesquisa (em números absolutos) 52 participantes, divididos em 10 enfermeiros (19,23\%), 17 técnicos de enfermagem $(32,70 \%)$ e 25 auxiliares de enfermagem $(48,07 \%)$. Dos 52 participantes, a maioria é do sexo feminino (84,62\%). Quanto ao estado civil, 63,46\% são casados ou estão em uma união estável e 36,54\% estão solteiros ou divorciados. Os respondentes possuem, em média, 1,42 filhos (com desvio padrão $(D P)=1,14)$. A maioria dos respondentes tem o ensino médio completo (48,07\%); os outros possuem o ensino superior incompleto $(19,23 \%)$ ou ensino superior completo $(32,70 \%)$. Quanto ao tipo de contrato de trabalho, 36,54\% dos respondentes são efetivos e $63,46 \%$ são contratados. Além disso, $48,07 \%$ possuem outro vínculo empregatício e $46,15 \%$ pensam em abandonar a profissão. Os respondentes possuem em média 13,38 anos $(\mathrm{DP}=8,18)$ de experiência profissional e trabalham no hospital, em média, há 6,76 anos $(\mathrm{DP}=6,88)$ e estão no setor de serviço atual, em média, há 3,98 anos $(\mathrm{DP}=5,26)$. Os enfermeiros atendem, em média, 30 pessoas por dia de trabalho, com uma variação de 5 a 150 pessoas por dia.

Os valores da média obtida nas três dimensões da $\mathrm{SB}$, assim como o desvio padrão, considerando a escala de 5 pontos, foram: para Exaustão Emocional (EE), média de 2,63 e $\mathrm{DP}=0,79$; para Despersonalização (DE), média de 2,0 com $\mathrm{DP}=$ 0,76 ; para Realização Profissional (RP), média de 3,79 com $\mathrm{DP}=0,66$.

A distribuição dos scores observados na amostra estudada em relação à Exaustão Emocional ficou 
configurada da seguinte forma: $50 \%$ dos participantes raramente se sentem exaustos emocionalmente, $25 \%$ se sentem algumas vezes exaustos emocionalmente e $7,7 \%$ frequentemente se sentem exaustos emocionalmente com o trabalho que realizam.

Em relação à frequência da manifestação da Despersonalização, verificou-se que $50 \%$ afirmaram que nunca vivenciaram essa dimensão, $36,54 \%$ afirmaram que raramente, $11,54 \%$ afirmaram que algumas vezes, e $1,92 \%$ afirmaram que frequentemente vivenciam essa dimensão. Isso significa que a maioria dos participantes declara não vivenciar a Despersonalização $(86,54 \%)$.

Sobre a dimensão Realização Profissional, a maioria dos participantes da pesquisa afirmou que algumas vezes $(46,15 \%)$ e frequentemente $(44,24 \%)$ sentem-se realizados profissionalmente, ou seja, $88,39 \%$ afirmam que se realizam com o trabalho. A minoria afirmou que nunca $(1,92 \%)$ e raramente $(5,77 \%)$ se sente realizada profissionalmente. Apenas $1,92 \%$ afirmaram sempre se sentirem realizados profissionalmente.

Ao investigar sobre a satisfação no trabalho, observou-se que os valores da média e o desvio padrão obtidos nos três fatores, considerando a escala de 5 pontos, foram: para satisfação com as relações hierárquicas (SRH), a média foi de 3,06 com $\mathrm{DP}=0,98$; para satisfação com o ambiente físico de trabalho (SAFT) a média foi de 2,58 com $\mathrm{DP}=1,04$; para satisfação intrínseca com o trabalho e oportunidades de crescimento (SITOPC), a média foi de 3,57 com $\mathrm{DP}=0,91$.

Sobre a SRH verificou-se que $15 \%$ dos participantes estão totalmente insatisfeitos, 25\% estão na maior parte das vezes insatisfeitos, $40,39 \%$ não estão nem satisfeitos, nem insatisfeitos, $15,38 \%$ estão satisfeitos na maior parte das vezes e apenas $3,84 \%$ dos respondentes afirmaram estar totalmente satisfeitos. Nesse fator, os respondentes avaliaram a igualdade de tratamento e sentimento de justiça, a participação nas decisões da instituição e do setor de trabalho, a forma como são avaliados e julgados em relação ao seu trabalho e o apoio que recebem dos superiores.

Já em relação ao SAFT, os resultados demonstraram que a maioria dos participantes afirmou estar totalmente insatisfeito $(25 \%)$ e insatisfeito na maior parte das vezes (36,54\%); 26,92\% afirmaram não estarem nem satisfeitos, nem insatisfeitos. Apenas 9,26\% afirmaram estar satisfeitos na maior parte das vezes e $1,92 \%$ afirmaram estar totalmente satisfeitos com o ambiente físico de trabalho. A avaliação da SAFT envolve os seguintes aspectos: espaço físico, iluminação, ventilação, temperatura e higiene e salubridade.

No último fator da satisfação, a SITOPC, foi observado que $3,84 \%$ dos participantes sentemse totalmente insatisfeitos, $19,23 \%$ sentem-se insatisfeitos na maior parte das vezes, $28,83 \%$ não se sentem nem satisfeitos, nem insatisfeitos, $44,24 \%$ sentem-se satisfeitos na maior parte das vezes e $3,84 \%$ sentem-se totalmente satisfeitos. Os elementos avaliados nesse fator dizem respeito à forma como o trabalhador se sente ao desempenhar o trabalho, ao conhecimento das metas e objetivos específicos em relação ao trabalho e à possibilidade de desenvolver trabalhos nos quais tem a possibilidade de se destacar.

Para avaliar a relação entre $\mathrm{SB}$ e satisfação no trabalho foi utilizada a regressão linear múltipla dos MQO. Considerando o número de participantes e de parâmetros a serem estimados, cada regressão possui 39 graus de liberdade, o que é suficiente para a validade do Teorema do Limite Central (TCL) (Casella \& Berger, 2010). Em outras palavras, a distribuição amostral tende a convergir para uma distribuição normal. Assim, de acordo com o TLC, a composição da amostra por 52 participantes é adequada para a realização de inferências estatísticas, possibilitando a implementação do modelo de regressão.

Os resultados encontrados estão nas Tabelas 1, 2 e 3.

De acordo com a Tabela 1, a análise de regressão linear múltipla demonstrou que a SAFT $(-0,489, \mathrm{p}=$ $<0,05)$ e a $\operatorname{SITOPC}(-0,870, \mathrm{p}=0,001)$ exercem influência negativa e estatisticamente significativa na Exaustão Emocional (EE), enquanto que a SRH $(0,004, p>0,05)$ não é estatisticamente significativa, ou seja, não se pode afirmar que essa variável exerce influência sobre a EE. As outras variáveis analisadas em conjunto, tais como número de filhos, tempo de experiência e trabalhar em outro local, não mostraram ser estatisticamente significantes para explicar a variação nos valores da EE. Esse modelo é explicado em $60,7 \%$ incluindo todas as variáveis $(\mathrm{F}(3,48)=15,90)$.

Na Tabela 2, a análise de regressão linear múltipla mostrou que, dos fatores de satisfação, somente o fator SITOPC $(-0,443, p<0,01)$ exerce influência negativa sobre a Despersonalização (DE). O tipo de contrato de trabalho (enfermeiro contratado) $(0,043, p>0,05)$ mostrou exercer influência na $\mathrm{DE}$. As outras variáveis de controle não se mostraram estatisticamente significantes. O coeficiente para determinação desse modelo é de 50,2\% $(F(3,48)=9,72)$. 
Tabela 1. Efeito das dimensões de satisfação no trabalho na dimensão Exaustão Emocional da Síndrome de Burnout pelo Método de Regressão Linear MQO $(\mathrm{n}=52)$

Variável dependente: Exaustão Emocional (EE)

Variável independente: Dimensões de Satisfação no Trabalho: Satisfação com as Relações Hierárquicas - SRH; Satisfação com o Ambiente Físico de Trabalho - SAFT; Satisfação Intrínseca com o Trabalho e Oportunidades de Crescimento - SITOPC.

\begin{tabular}{lcc}
\hline \multirow{2}{*}{ Variável Independente } & \multicolumn{2}{c}{ MQO } \\
\cline { 2 - 3 } & Coeficiente $\boldsymbol{\beta}$ & p-valor \\
\hline SRH & 0,004 & 0,967 \\
SAFT & $-0,489$ & $\mathbf{0 , 0 2 2} * *$ \\
SITOPC & $-0,870$ & $\mathbf{0 , 0 0 1} * * *$ \\
Idade & $-0,300$ & 0,049 \\
Feminino & 0,674 & 0,788 \\
Solteiro/Separado & 0,354 & 0,836 \\
Número de filhos & 0,027 & 0,974 \\
Enfermeiro efetivo & $-0,769$ & 0,739 \\
Enfermeiro contratado & $-2,299$ & 0,326 \\
Tempo de experiência & 0,004 & 0,972 \\
Tempo experiência no setor & 0,048 & 0,811 \\
Trabalha em outro local & 1,769 & 0,262 \\
\hline $\mathrm{F}(3,48)=15,90 ; \mathrm{R}^{2}=0,6071$ & & \\
\hline$* * * \mathrm{p}>0,01 ; * * \mathrm{p}>0,05$ & &
\end{tabular}

Tabela 2. Efeito das dimensões de satisfação no trabalho na dimensão Despersonalização da Síndrome de Burnout pelo Método de Regressão Linear MQO (n=52)

Variável dependente: Despersonalização (DE)

Variável independente: Dimensões de Satisfação no Trabalho: Satisfação com as Relações Hierárquicas - SRH; Satisfação com o Ambiente Físico de Trabalho - SAFT; Satisfação Intrínseca com o Trabalho e Oportunidades de Crescimento - SITOPC.

\begin{tabular}{|c|c|c|}
\hline \multirow{2}{*}{ Variável Independente } & \multicolumn{2}{|c|}{ MQO } \\
\hline & Coeficiente $\beta$ & p-valor \\
\hline SRH & $-0,120$ & 0,050 \\
\hline SAFT & $-0,009$ & 0,937 \\
\hline SITOPC & $-0,443$ & $0,004 * * *$ \\
\hline Idade & $-0,053$ & 0,545 \\
\hline Feminino & 0,781 & 0,602 \\
\hline Solteiro/Separado & $-0,129$ & 0,899 \\
\hline Número de filhos & 0,121 & 0,813 \\
\hline Enfermeiro efetivo & 1,994 & 0,151 \\
\hline Enfermeiro contratado & 2,866 & $0,043 * *$ \\
\hline Tempo de experiência & $-0,053$ & 0,474 \\
\hline Tempo experiência no setor & 0,234 & 0,059 \\
\hline Trabalha em outro local & 0,509 & 0,584 \\
\hline \multicolumn{3}{|l|}{$\mathrm{F}(3,48)=9,72 ; \mathrm{R}^{2}=0,5026$} \\
\hline \multicolumn{3}{|l|}{$* * * \mathrm{p}>0,01 ; * * \mathrm{p}>0,05$} \\
\hline \multicolumn{3}{|c|}{$\begin{array}{ll}\text { Na Tabela } 3 \text { verifica-se que a variável SITOPC } & \text { De acordo com os dados obtidos pelas } \\
(0,566, \mathrm{p}<0,05) \text { exerce influência na RP. Nesse caso } & \text { regressões, os fatores de satisfação no trabalho estão } \\
\text { também as outras variáveis analisadas não foram } & \text { associadas às dimensões da Síndrome de Burnout } \\
\text { estatisticamente significantes. As variáveis analisadas } & \text { em profissionais de enfermagem participantes desta } \\
\text { explicam } 29,6 \% \text { do modelo }(\mathrm{F}(3,48)=10,02) . & \text { pesquisa. }\end{array}$} \\
\hline
\end{tabular}


Tabela 3. Efeito das dimensões de satisfação no trabalho na dimensão Realização Profissional da Síndrome de Burnout pelo Método de Regressão Linear MQO (n=52)

Variável dependente: Realização Profissional (RP)

Variável independente: Dimensões de Satisfação no Trabalho: Satisfação com as Relações Hierárquicas - SRH; Satisfação com o Ambiente Físico de Trabalho - SAFT; Satisfação Intrínseca com o Trabalho e Oportunidades de Crescimento - SITOPC

\begin{tabular}{lcc}
\hline \multirow{2}{*}{ Variável Independente } & \multicolumn{2}{c}{ MQO } \\
\cline { 2 - 3 } & Coeficiente $\boldsymbol{\beta}$ & p-valor \\
\hline SRH & 0,089 & 0,318 \\
SAFT & 0,141 & 0,432 \\
SITOPC & 0,566 & $\mathbf{0 , 0 1 0 * * *}$ \\
Idade & 0,241 & 0,067 \\
Feminino & $-0,601$ & 0,782 \\
Solteiro/Separado & 1,225 & 0,412 \\
Número de filhos & $-0,267$ & 0,719 \\
Enfermeiro efetivo & $-0,888$ & 0,656 \\
Enfermeiro contratado & 0,190 & 0,925 \\
Tempo de experiência & $-0,126$ & 0,250 \\
Tempo experiência no setor & $-0,106$ & 0,546 \\
Trabalha em outro local & $-0,518$ & 0,702 \\
\hline $\mathrm{F}(3,48)=10,02 ; \mathrm{R}^{2}=0,2963$ & & \\
\hline
\end{tabular}

$* * * \mathrm{p}>0,01 ; * * \mathrm{p}>0,05$

\section{Discussão}

A presente pesquisa teve como foco a categoria profissional de enfermagem. Observa-se que a população estudada é composta em sua grande maioria por mulheres, dado encontrado em outros estudos que também têm como foco essa categoria profissional (Gil-Monte, 2002; Moreira et al., 2009). Essa realidade está relacionada ao processo histórico de constituição da profissão de enfermagem, que originalmente foi concebida como uma atividade caritativa religiosa realizada por mulheres (Elias \& Navarro, 2006; Gil-Monte, 2002).

No conjunto da população estudada também se verificou que a maioria possui outro vínculo empregatício, o que significa que esses indivíduos podem estar trabalhando 80 horas por semana. Essa jornada de trabalho excessiva tem impacto no tipo de assistência prestada e também contribui para o desenvolvimento da Síndrome de Burnout (Maslach, 2005; Wright \& Bonett, 1997) em algum momento da carreira.

Os dados sobre a SB apresentaram baixos índices de Exaustão Emocional (EE) e Despersonalização (DE) e relativamente altos índices de Realização Profissional (RP), contudo observam-se alguns indivíduos com índices que apresentam certa vulnerabilidade a síndrome. Essa realidade também é significativa uma vez que a SB pode ser disseminada por meio das interações sociais do trabalho (Maslach et al., 2001).

Os resultados encontrados sobre a satisfação no trabalho revelam níveis elevados de insatisfação em relação ao ambiente físico do trabalho $(61,54 \%)$ e razoavelmente elevados para a insatisfação em relação às relações hierárquicas $(40,38 \%)$. Já o índice sobre a satisfação com o trabalho e as oportunidades profissionais foi o mais elevado entre os fatores de satisfação pesquisados, uma vez que $48,08 \%$ afirmam estar satisfeitos na maior parte das vezes ou totalmente satisfeitos.

Esse conjunto de dados apresenta o local de trabalho pesquisado. $\mathrm{O}$ ambiente hospitalar é reconhecido como um ambiente insalubre, penoso e perigoso e, por isso, local privilegiado para o adoecimento (Elias \& Navarro, 2006). Acrescido a isso, condições de trabalho precárias como espaço físico limitado e não climatização adequada podem influenciar na forma como os enfermeiros avaliam o seu local de trabalho. As relações hierárquicas avaliadas refletem uma estrutura organizacional tecnoburocrática na qual existem, muito provavelmente, relações hierárquicas rígidas e autocráticas. Além disso, é possível que na 
visão dos respondentes não existam critérios claros em relação à delimitação de recompensas e punições. Apesar da avaliação negativa nesses dois itens, verifica-se que boa parte dos respondentes sente-se satisfeita com o trabalho que executa.

Foi analisada a relação entre as dimensões da SB e os fatores de satisfação por meio da técnica de análise de regressão linear múltipla MQO. Os resultados indicam relações significativas entre as dimensões da $\mathrm{SB}$ e os fatores de satisfação. Esses dados corroboram estudos anteriores que indicam que a Síndrome de Burnout ocorre como uma resposta crônica a questões referentes ao ambiente laboral (Benevides-Pereira, 2002; Silva et al., 2006). Os dados avaliados dizem respeito a três fatores relacionados à satisfação no ambiente do trabalho. São eles: satisfação com as relações hierárquicas, com o ambiente físico do trabalho e com o trabalho em si e as oportunidades de crescimento.

Em relação à satisfação com as relações hierárquicas, verifica-se que não há correlação significativa com o desenvolvimento da SB. Esse dado merece maiores estudos, uma vez que a satisfação com as relações hierárquicas (Silva \& Carlotto, 2008) e a satisfação com a chefia (Neves, 2012) geralmente contribui para a incidência da SB.

Sobre o fator ambiente físico de trabalho, os resultados demonstram que quanto menor é a satisfação em relação a essa questão, maior é o desenvolvimento de Exaustão Emocional. O resultado encontrado demonstra que outros elementos, além da sobrecarga de trabalho e do conflito pessoal (Maslach, 2005; Wright \& Bonett, 1997), podem interferir no desenvolvimento da EE, que são as condições físicas de trabalho. Os dados encontrados corroboram a literatura recente, já que o ambiente de trabalho é cada vez mais reconhecido pelas ciências da saúde como um dos fatores determinantes da saúde dos trabalhadores (Laranjeira, 2009).

O outro fator avaliado foi a satisfação com o trabalho e oportunidades de crescimento. Verificase que quanto menor a satisfação nesse fator, maior é a probabilidade de desenvolvimento de Exaustão Emocional e de Despersonalização e menor é a possibilidade de o indivíduo sentir-se realizado profissionalmente. Esse dado revela que esse fator interfere nas três dimensões da $\mathrm{SB}$ de maneira significativa. No contexto atual, o trabalhoé considerado como um espaço social de grande relevância para vida do indivíduo, uma vez que contribui em grande parte com a formação da identidade do indivíduo, assim como com as maneiras de interação entre as pessoas (Coutinho, Krawulski, \& Soares, 2007). Diante disso, a forma como o trabalho está estruturado em termos de tipo de tarefa, autonomia de trabalho e possibilidades de desenvolvimento impacta diretamente sobre o indivíduo. O trabalho pode ser, então, tanto um lugar de prazer quanto de sofrimento (Dejours, 1999). No caso da vivência do sofrimento no trabalho, a SB é uma das doenças ocupacionais que pode manifestar-se.

Verificou-se também que o tipo de trabalho impacta na dimensão Despersonalização, ou seja, aqueles que são contratados apresentam maior índice de DE. É possível que esses indivíduos mantenham contrato com outras instituições e o excesso de trabalho esteja contribuindo com a DE, já que o distanciamento é uma reação imediata à exaustão (Maslach et al., 2001).

\section{Considerações finais}

Este artigo teve como objetivo analisar a relação entre os fatores de satisfação no trabalho e à Síndrome de Burnout em profissionais de enfermagem de um hospital público do Sudeste. Os achados indicam que as dimensões de satisfação no trabalho (satisfação com o ambiente físico do trabalho e satisfação intrínseca com o trabalho e oportunidades de crescimento) impactam nas dimensões da Síndrome de Burnout (Exaustão Emocional, Despersonalização e Realização Profissional). Além dessas variáveis de satisfação, verificou-se que a variável tipo de trabalho também impacta na dimensão Despersonalização. Esses dados reforçam que o desenvolvimento da SB está relacionado ao trabalho (Maslach \& Schaufeli, 1993).

A partir dos resultados encontrados, podese considerar que políticas de mudança do contexto organizacional podem interferir no número de ocorrência da Síndrome de Burnout. Nesse sentido, é relevante reorganizar o trabalho dos profissionais de enfermagem de forma que seja pautado pela criação de ambientes físicos seguros e compatíveis com o tipo de trabalho desenvolvido; jornada de trabalho adequada; e definição clara e transparente de objetivos e metas, permitindo com isso que o profissional de enfermagem possa alcançá-las como meio de crescimento e de reconhecimento profissional. Em outras palavras, os resultados ajudam na determinação de políticas da gestão de pessoas na área de enfermagem, apontando os principais fatores que podem ser utilizados para que os problemas gerados com a Síndrome de Burnout sejam evitados ou pelo menos reduzidos.

Os resultados desta pesquisa dizem respeito a uma instituição específica e generalizações devem ser realizadas com cautela. Ressalta-se a necessidade 
de realizar novos estudos que avaliem quais ações são mais eficientes para aumentar os índices de satisfação no trabalho e, consequentemente, diminuir a ocorrência da Síndrome de Burnout em profissionais de enfermagem.

\section{Referências}

Benevides-Pereira, A. M. T. (2001). MBI - Maslach Burnout Inventory e suas adaptações para o Brasil [Resumo]. In XXXI Reunião Anual de Psicologia (pp. 84-85). Rio de Janeiro: SBP. Acesso em 25 de janeiro, 2010, em http://www. sbponline.org.br/resources/anais/2001.pdf

Benevides-Pereira, A. M. T. (2002). Burnout: quando o trabalho ameaça o bem estar do trabalhador. São Paulo: Casa do Psicólogo.

Benevides-Pereira, A. M. T. (2003). O estado da arte do Burnout no Brasil. Revista Eletrônica InterAção Psy, 1(1), 4-11. Acesso em 12 de março, 2011, em http:// www.saudeetrabalho.com.br/download_2/burnout-profuniversitario.pdf

Carlotto, M. S. (2002). A síndrome de burnout e o trabalho docente. Psicologia em Estudo, 7(1), 21-29.

Carlotto, M. S. \& Câmara, S. G. (2007). Propriedades psicométricas do Maslach Burnout Inventory em uma amostra multifuncional. Estudos de Psicologia (Campinas), 24(3), 325-332.

Carlotto, M. S. \& Câmara, S. G. (2008a). Análise da produção científica sobre a Síndrome de Burnout no Brasil. Psico, 39(2), 152-158.

Carlotto, M. S. \& Câmara, S. G. (2008b). Propriedades psicométricas do questionário de satisfação no trabalho (S20/23). PsicoUSF, 12(2), 203-210.

Casella, G. \& Berger, R. (2010). Inferência estatística. São Paulo: Centage Learning.

Coutinho, M. C., Krawulski, E., \& Soares, D. H. P. (2007). Identidade e trabalho na contemporaneidade: repensando articulações possíveis. Psicologia \& Sociedade 19(n. spe. 1), 29-37.

Cozby, P. C. (2003). Métodos de pesquisa em ciências do comportamento. São Paulo: Atlas.

Dejours, C. (1999). A banalização da injustiça social. Rio de Janeiro: Fundação Getúlio Vargas.

Dias, S. M. M., Boas, A. A. V., Dias, M. R. G, \& Barcellos K. C. P. (2005). Fatores desmotivacionais ocasionados pelo estresse de enfermeiros em ambiente hospitalar. In FEAUSP (Org.), VIII SEMEAD - Seminários de Administração FEA-USP (pp. 1-13). São Paulo: FEA-USP.

Elias, M. A. \& Navarro, V. L. (2006). A relação entre o trabalho, a saúde e as condições de vida: negatividade e positividade no trabalho das profissionais de enfermagem de um hospital escola. Revista Latino-Americana de Enfermagem, 14(4), 517-525.

Gil-Monte, P. R. (2001). El síndrome de quemarse por el trabajo (síndrome de Burnout): aproximaciones teóricas para su explicación y recomendaciones para la intervención. Revista PsicologiaCientifica.com, 3(5). Acesso em 25 de janeiro, 2010, em http://www.ucipfg.com/Repositorio/MSCG/ MSCG-16/BLOQUE-ACADEMICO/Unidad-2/lecturas/ psicologia-el-sindrome-de-quemarse-por-el-trabajo$\% 28$ sindrome-de-burnout $\% 29$-aproximaciones-teor.pdf
Gil-Monte, P. R. (2002). Influencia del género sobre el proceso de desarrollo del síndrome quemarse por el trabajo (burnout) en profesionales de enfermería. Psicologia em Estudo, 7(1), 3-10.

Laranjeira, C. A. (2009). O contexto organizacional e a experiência de estress: uma perspectiva integrativa. Revista de Salud Pública, 11(1), 123-133.

Maslach, C. (2005). Entendendo o Burnout. In A. M. Rossi, P. L. Perrewé, \& S. L. Sauter (Eds.), Stress e qualidade de vida no trabalho: perspectivas atuais da saúde ocupacional (pp. 122-148). São Paulo: Atlas.

Maslach, C. \& Jackson, S. E. (1981). The measurement of experienced burnout. Journal of Occupational Behavior, 2(2), 99-113.

Maslach, C. \& Schaufeli, W. B. (1993). The future of Burnout. In W. B. Schaufeli, C. Maslach, \& T. Marek (Eds.), Professional burnout: Recent developments in theory and research (pp. 253-259). New York: Taylor \& Francis.

Maslach, C., Schaufeli, W. B., \& Leiter, M. P. (2001). Job burnout. Annual Review of Psychology, 52, 397-422.

Meliá, J. L. \& Peiró, J. M. (1989). La medida de la satisfacción laboral en contextos organizacionales: El Cuestionario de Satisfacción S20/23. Psicologemas, 5, 59-74.

Moreira, D. S., Magnago, R. F., Sakae, T. M., \& Magalewski, F. R. L. (2009). Prevalência da síndrome de burnout em trabalhadores de enfermagem de um hospital de grande porte da Região Sul do Brasil. Cadernos de Saúde Pública, 25(7), 1559-1568.

Murofurose, T. N., Abranches, S. S., \& Napoleão, A. A. (2005). Reflexões sobre estresse e burnout e a relação com a enfermagem. Revista Latino-Americana de Enfermagem, 13(2), 255-261.

Neves, V. F. (2012). Impacto da satisfação no trabalho e da percepção de suporte organizacional sobre a Síndrome de Burnout em trabalhadores de enfermagem de um Hospital Universitário. Dissertação de Mestrado, Programa de Pós-Graduação em Psicologia, Universidade Federal de Uberlândia, Uberlândia, MG.

Oliveira, P. R., Tristão, R. M., \& Neiva, E. R. (2006). Burnout e suporte organizacional em profissionais de UTI-Neonatal. Educação Profissional: Ciência e Tecnologia, 1(1), 27-37.

Rosa, C. \& Carlotto, M. S. (2005). Síndrome de burnout e satisfação no trabalho em profissionais de uma instituição hospitalar. Revista SBPH, 8(2), 1-15.

Ruviaro, M. F. S. \& Bardagi, M. P. (2010). Síndrome de Burnout e satisfação no trabalho em profissionais da área de enfermagem do interior do RS. Barbarói, 33, 194-216.

Schaufeli, W. B. \& Taris, T. W. (2005). The conceptualization and measurement of burnout: Common ground and worlds apart. Work \& Stress, 19(3), 256-262.

Silva, B. M., Lima, F. R. F., Farias, F. S. A. B, \& Campos, A. C. S. (2006). Jornada de trabalho: fator que interfere na qualidade da assistência de enfermagem. Texto e Contexto Enfermagem, 15(3), 442-448.

Silva, T. D. \& Carlotto, M. S. (2008). Síndrome de burnout em trabalhadores da enfermagem de um hospital geral. Revista SBPH, 11(1), 113-130.

Stacciarini, J. M. R. \& Tróccoli, B. T. (2001). O estresse na atividade ocupacional de enfermeiro. Revista LatinoAmericana de Enfermagem, 9(2), 17-25.

Tamayo, M. R. (2009). Burnout: implicações das fontes organizacionais de desajuste indivíduo-trabalho em 
profissionais de enfermagem. Psicologia: Reflexão $e$ Crítica, 22(3), 474-482.

Wright, T. A. \& Bonett, D. G. (1997). The contribuition of burnout to work performance. Journal of Organizational Behavior, 18(5), 491-499.

\section{Agradecimento}

À FAPES e ao CNPQ pelo apoio e fomento. CNPq: Edital Universal, processo número 471873/2012-8 e FAPES: Edital Universal, processo número 54692075/11.

Submissão em: 26/06/2012

Revisão em: 27/03/2013

Aceite em: 03/06/2013

Adriana Müller Saleme de Sá é graduada em Enfermagem pela Universidade Federal do Espírito Santo (1996) e

Mestre em Administração pela Fundação Instituto Capixaba de Pesq. em Contabilidade, Economia e Finanças (2011).
Atualmente é professora da Universidade Vila Velha-UVV. Endereço: Avenida Comissário José Dantas de Melo, $n^{\circ} 21$, Boa Vista. Vila Velha/ES, Brasil. CEP 29102-920.

E-mail: adriana.sa@uvv.br

Priscilla de Oliveira Martins-Silva é Doutora, mestre e graduada em Psicologia pela Universidade Federal do Espirito Santo. Atualmente é professora da Universidade

Federal do Espirito Santo. E-mail: priscillamartinssilva@gmail.com

Bruno Funchal é graduado em Economia pela Universidade Federal Fluminense, doutorado em Economia pela Fundação Getúlio Vargas - RJ e Pós-Doutorado pelo Instituto Nacional de Matemática Pura e Aplicada (IMPA).

Atualmente é Professor Titular da FUCAPE Business School. E-mail: bfunchal@,fucape.br

\section{Como citar:}

Sá, A. M. S., Martins-Silva, P. O., \& Funchal, B. (2014). Burnout: o impacto da satisfação no trabalho em profissionais de enfermagem. Psicologia \& Sociedade, 26(3), 664-674. 